\title{
Rib 11
}

National Cancer Institute

\section{Source}

National Cancer Institute. Rib 11. NCI Thesaurus. Code C52768.

The eleventh rib counting from the top of the rib cage down. 